\title{
Research on DTX Technology and Power Consumption Performance of Mobile Communication Terminal
}

\author{
Shui Zhen XIE ${ }^{1, a}$ \\ ${ }^{1}$ School of electronic engineering, Jiujiang University, Jiujiang 330025, China
}

\begin{abstract}
In order to reduce the power consumption of GSM and TD-SCDMA mobile communication system terminal, the paper starts with DTX (Discontinuous Transmission) technology of GSM and TD-SCDMA systems, offers a detailed analysis of the DTX's function in optimizing power consumption of GSM and TDSCDMA mobile communication system terminal and reducing system interference, and verifies DTX's positive role in reducing the power consumption of the mobile terminal by experiment.
\end{abstract}

Key Words. Mobile terminal, green energy and energy conservation, power consumption performance, DTX

\section{Submitting the Manuscript}

Green energy, energy conservation and environmental protection has become a social consensus, awareness of energy conservation and emission reduction has been firmly established, as well as universal access to smart mobile phones and fierce increase of communications traffic, energy conservation of the communications industry allows of no delay. The mobile phones especially smart phones are closely related to people's daily work, life and entertainment, of which the standby time, power consumption, and recycling of used batteries, reflects the concept of green communications directly or indirectly. When people use mobile phones especially smart phones to experience with a range of voice, data, video and other multimedia services, the phone power consumption will be very fast, there will be insufficient of power supply of mobile phone batteries, resulting in frequent phone charging, which will consume more energy. If the performance of mobile phone is poor, frequent use and charging of mobile phones may make power battery hot, which affects the user's experience, even human health. In short, energy saving of mobile phone battery is the key protection to green communication, is the core of a low-carbon economy. Phone energy saving technologies, energy saving program has important practical significance for the construction of green communications and satisfaction improvement of mobile users.

\section{Main Factors Affecting Power Consumption Performance of Mobile Terminal}

The internal factors of mobile terminal, including: (1) battery performance of the mobile terminal; (2) power consumption of wireless transceiver (RF chipset); (3)power consumption and process characteristics of baseband chip; 4) peripheral devices power consumption of mobile terminal, such as keyboard backlight, speakers, screen displays; (5) power controls and power management scheme of the mobile terminal, etc.

External factors related to network environment: (1) the mode of mobile terminal, connected mode (ie, work status) has more power consumption than the idle mode (ie, standby); (2) movement of the mobile terminal, more frequent the mobile terminal moving costs more power consumption; (3) the quality of wireless network; (4) wireless network environment; (5) protocol stack control; (6) the set of network parameter values, such as DRX parameters, DTX parameters, and various of registration timers. DRX, DTX determines the interval that mobile terminal wakes to monitor the paging channel message in the idle state, the long interval means that the mobile terminal wakes up less, which will extend the standby time of the terminal. These parameters have obvious effect for the standby power consumption of mobile terminal.

The enhancement and improvement of mobile terminal power consumption performance is not only closely related to the user experience, the quality of mobile terminal, but also closely related to the green

\footnotetext{
${ }^{\mathrm{a}}$ Corresponding author: xieshuizhen@126.com
} 
communication concept. In general, according to the user's daily habits, the application state of the mobile terminal can be divided into the work state and the idle state, the corresponding technical indicators are operating current and standby current which measure overall power consumption performance of the mobile terminal. The power consumption of mobile terminal is relatively less in idle state, while it is more in the call state. For example, 6 TD Smart phone (TD smart phones), the power consumption in different states is shown in Figure 1. It can be seen in Figure 1, in the call state, TD terminal average power consumption is $140 \sim 160 \mathrm{~mA}$; in the idle state, TD terminal power consumption is at an average of $5 \sim 7 \mathrm{~mA}$. It is visible that power consumption in call state is much more than idle state. Thus, users are more concerned about how to reduce the operating current of the mobile terminal. There are a lot of factors affecting the power losses and operating current of mobile terminal, such as DTX period, re-election threshold, signal coverage strength, paging cycle, neighbor cell number, the paper researches DTX technology.

\section{DTX Technical Parameters and Introduction}

\subsection{Introduction of DTX Technical Features}

When users use mobile phone to call via mobile communication network, typically the two sides take turns to speak, that is, speech time of one side accounts for about $50 \%$ of the total communication time, the other time is silence period and listen to the other one talk, there is only background noise without voice signal during the silence. If all background noise information is transmitted to the mobile communication network, it will cost great waste of network system resources, and also increase interference within the network system. In order to reduce the total air emission levels in mobile communication system, improve mobile communication system efficiency and reduce the power consumption of wireless transmitter (especially mobile station), we use Voice Activity Detection (VAD) and Discontinuous transmission / comfortable noise generation (DTX / CNG) technology, discontinuous transmission / comfort noise generation (DTX / CNG) technology can encode in $13 \mathrm{~Kb}$ / s during a call, encode with $500 \mathrm{~b} / \mathrm{s}$ and send comfort noise in the standstill period, so that the average rate is lowered down. If it is a waste of channel resources that using the same bit rate to encode the voice signal, the discontinuous transmission / comfort noise generation (DTX / CNG) technology, can control encoders with different bit rate to transmit data in voice stage and background noise stage, which will reduce power consumption of wireless transmitter and a mobile station, while also reduce the total power of the wireless channel, so as to improve the channel utilization and reduce interference.

\subsection{The main control parameters}

DTXD: This parameter specifies whether the mobile station use DTX functionality in the downlink of the cell site. The application selection is functional for voice and non-transparent data, and functional for all $\mathrm{TCH}$ of all non-BCCH carrier within the cell. This parameter should be set on each cell.

DTXU: This parameter specifies whether the mobile station use DTX functionality in the uplink of the cell site. This parameter should be set in each cell.

$$
\begin{aligned}
& \text { DTXU }=1: \text { shall } \\
& \text { DTXU }=2: \text { shall not } \\
& \text { DTXU }=0: \text { may shall }
\end{aligned}
$$

\subsection{Special adjustable parameters}

DTXFUL: Define the number of measuring reports which selecting full set measurements requires.

\section{Test Method of GSM/TD-SCDMA Terminal Power Consumption}

This study tests in the current network environment, the test model is shown in Figure 2. The advantage of existing network environment is that the test environment itself is the actual working environment of the mobile terminal, test results of power consumption can directly reflect the power consumption performance of mobile terminal in use. The disadvantage is that it is difficult to control the wireless network system parameters, test results of power consumption are related to specific network parameters and wireless network environment. In this study, the test device comprises a plurality of GSM / TD-SCDMA dual-mode mobile terminal and a synthetical terminal tester SP6010, etc. In order to simulate terminal power performance more accurately in various conditions, we used the following 3 network environment for testing: moving state, fixed good signal quality, fixed poor signal quality. Test indicators take average current of DTX Enable before and after in call state as the main basis for judgment.

\section{Test Results and Analysis of DTX Settings Affecting Terminal Power Consumption Performance}

Respectively in the TD-SCDMA, GSM mode, use test instrumentation (terminal tester SP6010) DTX Enable function before and after, test the average current that GSM / TD-SCDMA dual-mode mobile terminal initiates a voice call, wherein, for TD-SCDMA mode, when DTX Enable, SBGP $=40 \mathrm{~ms}$ (SBGP value is cycle of no data transmitted). Test results are respectively shown in Figure 3 and Figure 4.

The test results of the experiment above show that after enabling DTX the GSM mobile terminal can save almost half of the power, namely the use of DTX reduces the power consumption of $2 \mathrm{G}$ terminals significantly, 
optimizes user's experience feelings, and does not adversely affect mobile communication system performance. For TD-SCDMA mobile terminal, it has a similar conclusion, if the network enables DTX function and set reasonable data transmission interval (ie DTX cycle SBGP), it so can save nearly half of the power after enabling DTX, and will be a great help for terminal power saving and reducing the system interference.

\section{Summary}

Improving the performance of terminal power consumption has important practical significance for improving users' satisfaction and enhancing users' experience feelings. Through the practice research of the project, we can see the introduction of DTX technology to mobile communication network system can significantly reduce battery consumption of mobile terminals, reduce wireless system interference, application of DTX can greatly reduce the power consumption of the mobile station. At the same time, through continuously optimizing and improving DTX technology, it can improve the flexibility and controllability in various scenarios, which has a very important practical significance for strengthening the green channel idea of mobile communication system and enhancing the user experience.
Note: This paper is the research achievement of school level topic 'DTX technology application in mobile communication network' of Jiu Jiang school, topic number: 2014KJYB004.

\section{References}

1. Li Jiandong. Mobile Communications, Xi'an University of Electronic Science and Technology Press, 2006

2. Wang Shiliang. Research on power consumption performance of $3 \mathrm{G}$ mobile communication terminal, Mobile Communications, 2005.12.25,37-38

3. Liu Dongdong. Research on technology enhancing mobile voice quality (MOS), Master's degree thesis of Beijing University of Posts, 2012.9

4. Zhou Dejun. Discontinuous transmission technology in voice communication, Communication Technology, 2001(9), 46-48

5. Li Fangwei, Zhang Yaqing. Green energy technology of TD-SCDMA terminal, Communication Technology of Guangdong province, 2010.3

6. Dong Yanjie, Zhou Meng. Power consumption test methods and test results analysis of TD-SCDMA terminal, Telecommunications engineering technology and standardization, 2008.10

\section{Acknowledgment}

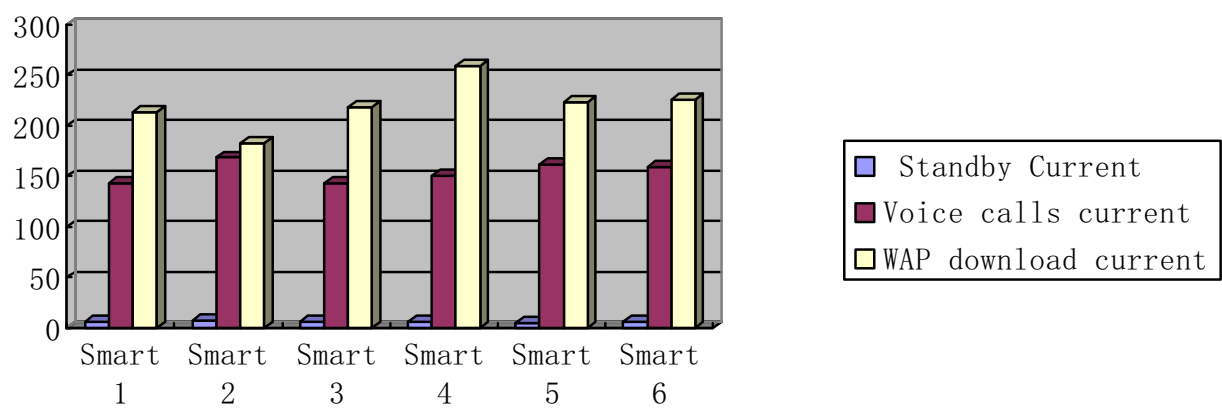

Figure 1. Test sample of TD terminal operating current and standby current (Current unit: $\mathrm{mA}$ ) 


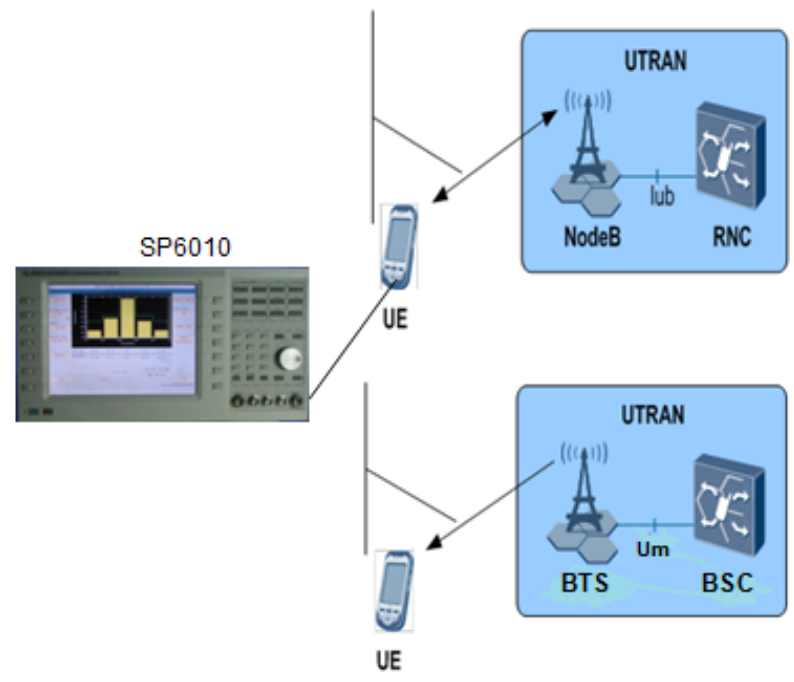

Figure 2. Test model of terminal power consumption

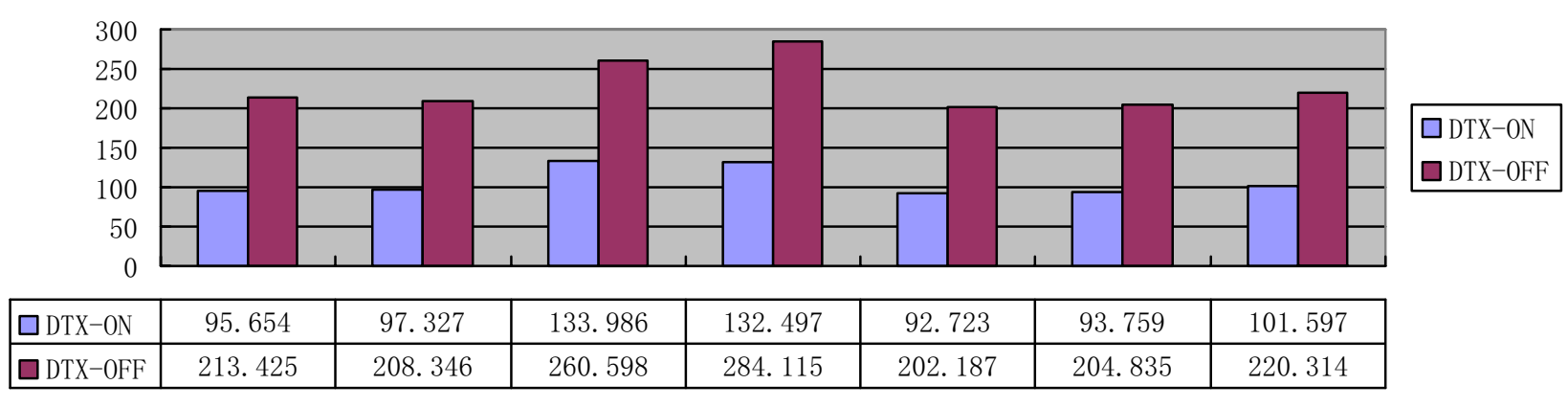

Figure 3. The impact of DTX Enable before and after to GSM terminal power consumption (current unit mA)

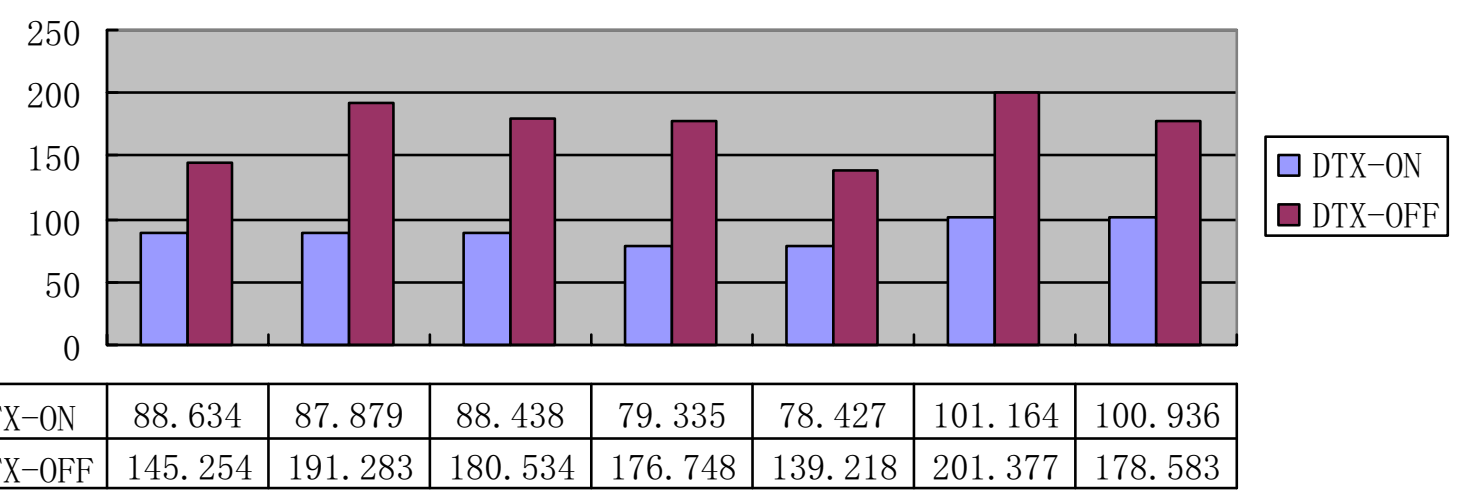

Figure 4. The impact of DTX Enable before and after to TD terminal power consumption (current unit mA) 